\title{
Multi-room occupancy estimation through adaptive gray-box models
}

\author{
A. Ebadat, G. Bottegal, M. Molinari, D. Varagnolo, \\ B. Wahlberg, Fellow, IEEE, H. Hjalmarsson, Fellow, IEEE, and K. H. Johansson, Fellow, IEEE
}

\begin{abstract}
We consider the problem of estimating the occupancy level in buildings using indirect information such as $\mathrm{CO}_{2}$ concentrations and ventilation levels. We assume that one of the rooms is temporarily equipped with a device measuring the occupancy. Using the collected data, we identify a gray-box model whose parameters carry information about the structural characteristics of the room. Exploiting the knowledge of the same type of structural characteristics of the other rooms in the building, we adjust the gray-box model to capture the $\mathrm{CO}_{2}$ dynamics of the other rooms. The occupancy estimators are then designed using a regularized deconvolution approach which aims at estimating the occupancy pattern that best explains the observed $\mathrm{CO}_{2}$ dynamics. We evaluate the proposed scheme through extensive simulation using a commercial software tool, IDA-ICE, for dynamic building simulation.

Index Terms-Occupancy estimation, Maximum Likelihood, $\mathrm{CO}_{2}$ dynamics, inference, building automation
\end{abstract}

\section{INTRODUCTION}

The estimation of occupancy levels in buildings has important implications in efficient control of Heating, Venting and Air Conditioning (HVAC) systems and diagnostics [1], [2], [3], [4], [5], [6], [7]. Instrumenting buildings with dedicated hardware such as camera systems may raise privacy concerns and be economically disadvantageous, in particular when this requires retrofitting old structures. On the other hand, there is an increasing interest in understanding the effectiveness of estimating occupancy using non-dedicated information sources in buildings, such as $\mathrm{CO}_{2}$ concentration and air inlet actuation levels.

There are two main strategies to estimate occupancy in buildings. The first utilizes direct occupancy measurements collected by people-counting devices (see [8], [9] for a survey). The second strategy exploits non-dedicated sensor and devices. The typical approach is to design occupancy estimators by inverting the $\mathrm{CO}_{2}$ dynamics. The model relating the $\mathrm{CO}_{2}$ concentration with occupancy can be derived either

A. Ebadat, G. Bottegal, M. Molinari, B. Wahlberg, H. Hjalmarsson and K. H. Johansson are with ACCESS and the Department of Automatic Control, School of Electrical engineering, KTH Royal Institute of Technology, Sweden. Emails: \{ ebadat | bottegal | marcomo | bo | hjalmars | kallej \}@kth.se. D. Varagnolo is with the Division of Signals and Systems, Department of Computer Science, Electrical and Space Engineering, Luleå University of Innovation and Technology, Luleå, Sweden. Email: damiano.varagnolo@ltu.se.

This work is supported by the European Institute of Technology (EIT) Information and Communication Technology (ICT) Labs, the Swedish Energy Agency, the Swedish Governmental Agency for Innovation Systems (VINNOVA), the Knut and Alice Wallenberg Foundation, the Framework Programme for Research and Innovation Horizon 2020 under grant agreement n. 636834 (DISIRE), and the Swedish research council Norrbottens Forskningsråd. using physics-based concepts (e.g., mass-balance equations) or by employing data-based modeling techniques. As for the physics-based $\mathrm{CO}_{2}$ models, assuming well-mixed air in the room, authors in [10] derived a bilinear model which has similarities with the model presented in this paper. Still assuming well-mixed air, [11], [12] and [5] make use of massbalance equations and linear models for the $\mathrm{CO}_{2}$ dynamics. More detailed models are considered in [13]. Regarding databased modeling techniques, [14] uses methods of moments, while [15] proposes both linear parametric and nonparametric identified models, from which estimators based on deconvolution are designed. A novel approach is proposed in [16], where, using blind system identification techniques as in [17], no training sets including occupancy measurements are required. Other types of estimators use black box models identified using, e.g., neural networks or hidden Markov models, and potentially including several sources of information (e.g., temperature, humidity, concentrations, door statuses and light status, sound and motion, electricity consumption patterns) [18], [19], [20], [21], [22], [23], [24], [25]. This literature focuses on occupancy estimation in single rooms. Besides a few studies dealing with modeling and estimation of occupancy movements across buildings (see e.g., [26], [19]), the multi-room case has not received as much attention as the single-room case.

In this paper, we take an important step towards the extension of single-room occupancy estimators to the multiroom case. Our fundamental question is whether the information on the $\mathrm{CO}_{2}$ dynamics gathered in one room can be exploited to design occupancy estimators for other rooms of the same building. To answer such a question, we assume that one room of the building is temporarily equipped with an occupancy measurement device. We use the data collected by this device, together with $\mathrm{CO}_{2}$ concentration and ventilation data, to identify a nonlinear gray-box model via Maximum Likelihood (ML) [27]. The structure of the gray-box model is derived from first principles [28] and it permits defining a one-to-one correspondence between the model parameter vector and the physical parameters characterizing the room (i.e., room volume and size of the ventilation system). Exploiting this correspondence, we adapt the gray-box model to the characteristics of the other rooms, and we design an occupancy estimation based on regularized environmental signal deconvolution, similarly to the strategy proposed in [15]. The role of regularization here is to promote piecewise constant occupancy patterns.

We evaluate the proposed estimation scheme on a simu- 
lated environment generated using the commercial software IDA-ICE [29]. The simulated environment models a building on the KTH campus. The generated data are validated by comparison with a dataset available on the [30].

The paper is structured as follows. In Section II, we derive and identify the physics-based gray-box model which models the $\mathrm{CO}_{2}$ dynamics. In Section III, we introduce the occupancy estimator based on the identified model. In Section IV we present the problem of extending the occupancy estimator to the multi-room case. Experiments are reported in Section V. Some conclusions end the paper.

\section{Modeling AND IDENTIFICATION OF THE $\mathrm{CO}_{2}$ DYNAMICS}

\section{A. A physics-based model}

The $\mathrm{CO}_{2}$ concentration of room $j$, denoted by $\bar{c}_{j}(t)$, can be modeled from mass-conservation considerations, assuming well-mixed air (see [28]):

$$
\begin{aligned}
v_{j} \frac{d \bar{c}_{j}(t)}{d t} & =\left(\dot{Q}_{j}^{\text {vent,sup }}+\dot{Q}_{j}^{\text {leak,in }}\right) c \\
& -\left(\dot{Q}_{j}^{\text {vent,exh }}+\dot{Q}_{j}^{\text {leak,out }}\right) \bar{c}_{j}(t)+g o_{j}(t) .
\end{aligned}
$$

Here, $v_{j}$ is the volume of the room, and $c$ is the outdoor air $\mathrm{CO}_{2}$ concentration, which we assume constant and equal to $420 \mathrm{ppm} ; \dot{Q}_{j}^{\text {vent,sup }}$ and $\dot{Q}_{j}^{\text {vent,exh }}$ represent the supply and exhaust mechanical ventilation rates and $\dot{Q}_{j}^{\text {leak,in }}$ and $\dot{Q}_{j}^{\text {leak,in }}$ the inflow and outflow air leakages through doors and windows. The term $g o_{j}(t)$ models the occupants $\mathrm{CO}_{2}$ generation in the room, where $g$ is the $\mathrm{CO}_{2}$ generation rate per person and $o_{j}(t)$ is the number of occupants at time $t$. In the case of balanced ventilation it is reasonable to assume that $\dot{Q}_{j}^{\text {vent,sup }} \approx \dot{Q}_{j}^{\text {vent }} \approx \dot{Q}_{j}^{\text {vent,exh }}$ and that $\dot{Q}_{j}^{\text {leak,in }} \approx \dot{Q}^{\text {leak }} \approx$ $\dot{Q}_{j}^{\text {leak,out }}$. Equation (1) can then be simplified to

$$
\frac{d \bar{c}_{j}(t)}{d t}=\frac{\dot{Q}_{j}^{\mathrm{vent}}}{v_{j}}\left(c-\bar{c}_{j}(t)\right)+\frac{\dot{Q}^{\mathrm{leak}}}{v_{j}}\left(c-\bar{c}_{j}(t)\right)+\frac{g}{v_{j}} o_{j}(t) .
$$

In the ventilation system considered in this work, there is a constant ventilation flow in the zones, which can be increased if the indoor $\mathrm{CO}_{2}$ concentration is above a certain threshold. Under these assumptions, (2) can be rewritten as

$$
\begin{aligned}
\frac{d \bar{c}_{j}(t)}{d t} & =\frac{\dot{Q}_{j}^{\mathrm{vent}, \mathrm{min}}+\left(\dot{Q}_{j}^{\mathrm{vent}, \mathrm{max}}-\dot{Q}_{j}^{\mathrm{vent}, \mathrm{min}}\right) u_{j}(t)}{v_{j}}\left(c-\bar{c}_{j}(t)\right) \\
& +\frac{\dot{Q}^{\text {leak }}}{v_{j}}\left(c-\bar{c}_{j}(t)\right)+\frac{g}{v_{j}} o_{j}(t) .
\end{aligned}
$$

Since $\dot{Q}_{j}^{\text {vent,min }}$ does not depend on the ventilation control signal $u_{j}(t)$, we rewrite (3) as

$$
\frac{d \bar{c}_{j}(t)}{d t}=\frac{\dot{Q}_{j}^{\mathrm{u}} u_{j}(t)}{v_{j}}\left(c-\bar{c}_{j}(t)\right)+\frac{\dot{Q}_{j}^{\mathrm{c}}}{v_{j}}\left(c-\bar{c}_{j}(t)\right)+\frac{g}{v_{j}} o_{j}(t)
$$

with $\dot{Q}_{j}^{\mathrm{u}}=\dot{Q}_{j}^{\mathrm{vent}, \mathrm{max}}-\dot{Q}_{j}^{\mathrm{vent}, \mathrm{min}}$ and $\dot{Q}_{j}^{\mathrm{c}}=\dot{Q}_{j}^{\mathrm{vent}, \mathrm{min}}+\dot{Q}^{\text {leak }}$.
We discretize the continuous-time model (4) using the backward Euler discretization ${ }^{1}$, so we obtain

$\frac{\bar{c}_{j}(k)-\bar{c}_{j}(k-1)}{T}=\frac{\left(\dot{Q}_{j}^{\mathrm{u}} u_{j}(k)+\dot{Q}_{j}^{\mathrm{c}}\right)}{v_{j}}\left(c-\bar{c}_{j}(k)\right)+\frac{g}{v_{j}} o_{j}(k)$,

where $T$ is the sampling time. We define $c_{j}(k):=\bar{c}_{j}(k)-c$ and the parameter vector $\boldsymbol{\theta}_{j}^{T}:=\left[\begin{array}{lll}\theta_{j}^{\prime} & \theta_{j}^{\prime \prime} & \theta_{j}^{\prime \prime \prime}\end{array}\right]$, where

$$
\left\{\begin{aligned}
\theta_{j}^{\prime} & :=\frac{v_{j}}{v_{j}+T \dot{Q}_{j}^{\mathrm{u}}} \\
\theta_{j}^{\prime \prime} & :=\frac{T g}{v_{j}+T \dot{Q}_{j}^{\mathrm{u}}} \\
\theta_{j}^{\prime \prime \prime} & :=\frac{T \dot{Q}_{j}^{\mathrm{c}}}{v_{j}+T \dot{Q}_{j}^{\mathrm{u}}}
\end{aligned}\right.
$$

We assume that the measurements of $c_{j}(k)$ are corrupted by additive noise. Then, the measured $\mathrm{CO}_{2}$ concentration, denoted by $y_{j}(k)$, can be expressed through the measurement model $y_{j}(k)=c_{j}(k)+e_{j}(k)$, where $e_{j}(k)$ is the noise, assumed white and Gaussian. The overall model for the $\mathrm{CO}_{2}$ dynamics can be rewritten as the nonlinear Output Error (OE) system

$$
\left\{\begin{array}{l}
c_{j}(k)=\frac{\theta_{j}^{\prime}}{1+\theta_{j}^{\prime \prime \prime} u_{j}(k)} c_{j}(k-1)+\frac{\theta_{j}^{\prime \prime}}{1+\theta_{j}^{\prime \prime \prime} u_{j}(k)} o_{j}(k) \\
y_{j}(k)=c_{j}(k)+e_{j}(k) .
\end{array}\right.
$$

\section{B. Identification of the gray-box model}

In this section we describe a procedure for identifying the parameter vector $\boldsymbol{\theta}_{j}$ characterizing the model (7). Here, we assume that we have collected the dataset of information from room $j$

$$
\mathcal{D}_{j}:=\left\{y_{j}(k), u_{j}(k), o_{j}(k)\right\}_{k \in \mathcal{K}_{j}},
$$

containing recorded occupancy levels plus environmental information from the building supervisory control and data acquisition (SCADA) system for a set of time indexes $\mathcal{K}_{j}$,

Let us introduce the auxiliary notation

$$
a_{j}(k):=\frac{\theta_{j}^{\prime}}{1+\theta_{j}^{\prime \prime \prime} u_{j}(k)}, \quad b_{j}(k):=\frac{\theta_{j}^{\prime \prime}}{1+\theta_{j}^{\prime \prime \prime} u_{j}(k)},
$$

so that (7) becomes

$$
c_{j}(k)=a_{j}(k) c_{j}(k-1)+b_{j}(k) o_{j}(k) .
$$

Expanding recursively (10) back in time, and defining the quantities

$$
\begin{gathered}
\widetilde{c}_{j}(k):=c_{j}(k)-c_{j}(0) \prod_{\tau=0}^{k-1} a_{j}(k-\tau), \\
B_{j}(k, k-h):=b_{j}(k-h) \prod_{\tau=0}^{h-1} a_{j}(k-\tau)
\end{gathered}
$$

\footnotetext{
${ }^{1}$ This choice is motivated by the fact that backward Euler discretization led to better identification and estimation performance than the forward Euler discretization.
} 
(with the convention that $\prod_{\tau=0}^{-1} \star=1$ for every possible $\star$ ), it follows that

$$
\left[\begin{array}{c}
\widetilde{c}_{j}(1) \\
\vdots \\
\widetilde{c}_{j}(k)
\end{array}\right]=\left[\begin{array}{ccc}
B_{j}(1,1) & & 0 \\
\vdots & \ddots & \\
B_{j}(k, 1) & \cdots & B_{j}(k, k)
\end{array}\right]\left[\begin{array}{c}
o_{j}(1) \\
\vdots \\
o_{j}(k)
\end{array}\right] .
$$

Given $u_{j}(1), \ldots, u_{j}(k), o_{j}(1), \ldots, o_{j}(k)$ and our Gaussian assumptions on the noise $e_{j}(k)$ in (7), we have that

$$
\begin{aligned}
\widehat{c}_{j}\left(k ; \boldsymbol{\theta}_{j}\right) & :=y_{j}(0) \prod_{\tau=0}^{k-1} a_{j}(k-\tau) \\
& +\left[\begin{array}{lll}
B_{j}(k, 1) & \cdots & B_{j}(k, k)
\end{array}\right]\left[\begin{array}{c}
o_{j}(1) \\
\vdots \\
o_{j}(k)
\end{array}\right],
\end{aligned}
$$

is the best estimator of $c_{j}(k)$ for the parameter guess $\boldsymbol{\theta}_{j}$. This estimator can then be used for defining the ML estimator for the parameters $\boldsymbol{\theta}_{j}$ given the dataset $\mathcal{D}_{j}$. The estimate $\widehat{\boldsymbol{\theta}}_{j}$ is obtained solving

$$
\widehat{\boldsymbol{\theta}}_{j}:=\arg \min _{\widetilde{\boldsymbol{\theta}}_{j} \in \mathbb{R}^{3}} \sum_{k \in \mathcal{K}_{j}}\left(y_{j}(k)-\widehat{c}_{j}\left(k ; \widetilde{\boldsymbol{\theta}}_{j}\right)\right)^{2} .
$$

Even if problem (15) is nonlinear, it involves only three decision variables and can be effciently solved using standard interior point methods [31].

\section{ESTIMATING OCCUPANCY LEVELS BY REGULARIZED DECONVOLUTION}

In this section we revise the occupancy estimation approach proposed in [15], with some modifications to adjust it to the nonlinear gray-box model (7). We assume that we have the estimate $\widehat{\boldsymbol{\theta}}_{j}$ of the parameters of room $j$, and that, for each time instant $k$, we have access to $y_{j}(k), y_{j}(k-1)$ and $u_{j}(k)$, but not to $o_{j}(k)$.

From the assumption of Gaussianity of the measurement noise $e_{j}(k)$ in (7), the best unbiased estimator of $o_{j}(k)$ corresponds to a Least Squares (LS) estimator. However, since we know that candidate occupancy patterns are piecewise constant, more effective estimators can be obtained by applying regularized estimators. Let us introduce the following matrix and vector notation

$$
\begin{gathered}
\widetilde{y}_{j}(k):=y_{j}(k)-y_{j}(0) \prod_{\tau=0}^{k-1} a_{j}(k-\tau) \\
\widetilde{\boldsymbol{y}}_{j}:=\left[\begin{array}{c}
\widetilde{y}_{j}(1) \\
\vdots \\
\widetilde{y}_{j}(k)
\end{array}\right], \quad \boldsymbol{o}_{j}:=\left[\begin{array}{c}
o_{j}(1) \\
\vdots \\
o_{j}(k)
\end{array}\right], \quad B_{j}:=\left[B_{j}(h, \tau)\right] .
\end{gathered}
$$

Furthermore, we introduce the discrete derivative of $o_{j}(k)$ as

$$
\begin{gathered}
\Delta o_{j}(\tau):=o_{j}(\tau)-o_{j}(\tau-1), \quad \tau=1, \ldots, k-1, \\
\Delta \boldsymbol{o}_{j}:=\left[\Delta o_{j}(1), \ldots, \Delta o_{j}(k-1)\right]
\end{gathered}
$$

In [15], the following estimator for occupancy was introduced:

$$
\widehat{\boldsymbol{o}}_{j}=\left\lfloor\arg \min _{\widetilde{\boldsymbol{o}}_{j} \in \mathbb{R}_{+}^{k}}\left\|\tilde{\boldsymbol{y}}_{j}-B_{j} \widetilde{\boldsymbol{o}}_{j}\right\|_{2}^{2}+\lambda_{j}\left\|\Delta \widetilde{\boldsymbol{o}}_{j}\right\|_{1}\right\rceil,
$$

where $\lfloor\cdot 7$ denotes the vector-wise rounding operator, which is used to obtain integer solutions. This estimator is composed of a LS-type part, which favors adherence to data, and a $\ell_{1}$ (component) depending on the derivative of the unknown occupancy. This latter component promotes piecewise constant solution patterns. The parameter $\lambda_{j}$ allows a trade-off between the two components. We refer to [15] for further details.

Problem (17) is usually called fused-lasso. More elaborated theoretical analysis on the performance of these estimators can be found in [32] and [33].

It is straightforward to modify (17) to obtain an online estimator which considers only a fixed-length (say, $N$ ) data window of the past. At each time instant, the estimator is run by constructing the vectors $\widetilde{\boldsymbol{y}}_{j}$ and $\boldsymbol{o}_{j}$ using the latest $N$ data of the past. The length $N$ is chosen so that the computational complexity is low enough to allow a real-time solution of (17) and so that the discarded information does not influence significantly the outcomes of the estimator. A reasonable choice for tuning $\lambda_{j}$ in (17) is to use the value $\widehat{\lambda}_{j}$ that leads to the best estimation performance on the dataset used for training the parameters $\widehat{\boldsymbol{\theta}}_{j}$ in Section II-B. The performance index can be chosen as

$$
\left\|\boldsymbol{o}_{j}-\hat{\boldsymbol{o}}_{j}\left(\lambda_{j}\right)\right\|_{2},
$$

where $\boldsymbol{o}_{j}$ is constructed from the dataset (8) and $\hat{\boldsymbol{o}}_{j}\left(\lambda_{j}\right)$ is the occupancy pattern obtained using $\lambda_{j}$ in the estimator (17).

\section{FROM SINGLE-ROOM TO MULTI-ROOMS ESTIMATORS}

This section is dedicated to the extension of the estimator (17) to a generic room in a building that is not instrumented with occupancy sensors, by exploiting the information on the $\mathrm{CO}_{2}$ dynamics obtained in one room of the same building which is instrumented with occupancy sensors. We assume that the sampling time $T$ and the volume $v_{j}$ are known for all the rooms of interest.

Assume that every single room in a generic building is instrumented with sensors measuring $\mathrm{CO}_{2}$ and HVAC actuation levels (which are generally available in standard HVAC systems). We also assume that only one of the rooms, denoted by $j=0$, is instrumented with occupancy sensors for a short period. For this room the dataset $\mathcal{D}_{0}$ defined in (8) is available and thus it is possible to identify the $\mathrm{CO}_{2}$ dynamic of the room by estimating the unknown parameters $\boldsymbol{\theta}_{0}$ through (15) and estimate the occupancy levels invoking estimator (17).

However, for the rooms without occupancy measurements, the estimator (15) cannot be used to find the $\mathrm{CO}_{2}$ dynamic due to the lack of training set. We call these rooms untrained rooms. Define $\mathcal{I}$ as the set of rooms without occupancy measurements or untrained rooms. The question is now how to extend the estimator (17) to these rooms. 
To implement the estimator (17) one needs to know the $\mathrm{CO}_{2}$ dynamics of the room, i.e., (7), or alternatively, $\boldsymbol{\theta}_{j}$ and the regularization parameter $\lambda_{j}$. Finding the variables $\boldsymbol{\theta}_{j}$ for a room, in turn, requires either a training set $\mathcal{D}_{j}$ or the knowledge of $\dot{Q}_{j}^{\mathrm{c}}, \dot{Q}_{j}^{\mathrm{u}}, g$ since there is a one-to-one correspondence between $\boldsymbol{\theta}_{j}$ and $\dot{Q}_{j}^{\mathrm{c}}, \dot{Q}_{j}^{\mathrm{u}}, g$. Since for the rooms $j \in \mathcal{I}$ the set $\mathcal{D}_{j}$ is not available, we need to infer the triplet $\left(\dot{Q}_{j}^{\mathrm{c}}, \dot{Q}_{j}^{\mathrm{u}}, g\right)$ and the regularization parameter $\lambda_{j}$ from the training room and other available information.

\section{A. Estimating $\left(\dot{Q}_{j}^{u}, \dot{Q}_{j}^{c}, g\right)$}

We pose the following assumptions

$$
\begin{aligned}
& \frac{\dot{Q}_{j}^{\text {vent,max }}}{\dot{Q}_{0}^{\text {vent,max }}}=\frac{M_{j}}{M_{0}}, \quad \forall j, \\
& \frac{\dot{Q}_{j}^{\text {vent,min }}}{\dot{Q}_{0}^{\text {vent,min }}}=\frac{M_{j}}{M_{0}}, \quad \forall j,
\end{aligned}
$$

where $M_{j}$ and $M_{0}$ are parameters proportional to the ventilation inlet area serving the rooms $j$ and the training room, respectively. According to the assumptions the maximum and minimum ventilation air flows are proportional to the total inlet area in a room and the parameters $M_{j}$ can be obtained easily by physical inspection of the rooms. However, the assumptions are made for the purpose of this paper and might not be always applicable. The main reason is that the ventilation system design also depends on the room usage. We will study the implications of these assumptions through a simulated example.

Due to the assumptions, we can write

$$
\begin{aligned}
& \dot{Q}_{j}^{\mathrm{u}}=M_{j} \dot{Q}^{\mathrm{u}}, \quad \forall j, \\
& \dot{Q}_{j}^{\mathrm{c}}=M_{j} \dot{Q}^{\mathrm{c}}, \quad \forall j,
\end{aligned}
$$

where $\dot{Q}^{\mathrm{u}}$ and $\dot{Q}^{\mathrm{c}}$ are constant values. For (19b) we used the fact that the value of $\dot{Q}^{\text {leak }}$ is negligible compared to $\dot{Q}_{j}^{\text {vent,min }}$ and thus $\dot{Q}_{j}^{\text {c }} \approx \dot{Q}_{j}^{\text {vent,min }}$. Based on (19), we can readapt the gray-box model of the training room to the characteristics of the other rooms. It is sufficient to estimate the triplet $\left(\dot{Q}_{j}^{\mathrm{c}}, \dot{Q}_{j}^{\mathrm{u}}, g\right)$ for one room to be able to find it for all rooms. In order to estimate the triplet $\left(\dot{Q}_{j}^{\mathrm{c}}, \dot{Q}_{j}^{\mathrm{u}}, g\right)$ for any room $j \in \mathcal{I}$, we can thus start from the training room and estimate the unknown parameters $\widehat{\theta}_{0}$ through the singleroom estimator (17). The parameters $\dot{Q}_{0}^{\mathrm{c}}, \dot{Q}_{0}^{\mathrm{u}}$ and $g$ can be obtained invoking (6).

Once the triplet $\left(\dot{Q}_{0}^{\mathrm{c}}, \dot{Q}_{0}^{\mathrm{u}}, g\right)$ is obtained, one can use the information on $M_{j}$ and $v_{j}$ together with (6) to find $\widehat{\theta}_{j}$ for all $j \in \mathcal{I}$ and therefore the $\mathrm{CO}_{2}$ dynamic of the untrained rooms. It is then straightforward to estimate the occupancy levels using the estimator (17).

\section{B. Estimating $\lambda_{j}$}

The regularization parameter $\lambda_{j}$ is connected to the usage of the room as well as its structural characteristics. For instance, if a crowd size in a room is changing frequently, then $\lambda_{j}$ should be small (and vice versa). The problem of generalizing $\lambda_{j}$ to untrained rooms is an open problem and cannot be answered without additional assumptions on the usage of the room. In the following we analyze two different cases, corresponding to two specific hypotheses on the usage patterns in buildings. Although the suggested strategies are not immediately applicable in practical situations, they can produce some basic ideas on the choice of $\lambda_{j}$.

1) Assuming the same usage pattern for the rooms: Assume $\lambda_{j}=\lambda$ for all $j$; in this case $\lambda$ should be estimated by coupling the tuning procedures described in Section III by finding the best $\hat{\lambda}$ in the occupancy estimator for the training room.

2) Assuming the usage patterns to depend on the size of the room: One may assume

$$
\lambda_{j}=\lambda v_{j}
$$

i.e., the usage pattern depends linearly on the size (for simplicity, the volume, assuming that the ceilings heights are equal among different rooms). This simple assumption leads to the strategy

$$
\frac{\lambda_{j}}{\lambda_{0}}=\frac{v_{j}}{v_{0}},
$$

where $\lambda_{0}$ is obtained by solving the tuning problem in Section III for the training room. Once $\lambda_{0}$ in (20) has been found, then generalizing to other untrained rooms is immediate, as soon as one knows the relative room volumes $v_{j}$.

\section{ASSESSING THE EXTENDED OCCUPANCY ESTIMATORS}

We evaluate the effectiveness of our derivations, through a building simulations tool. The dedicated experiments are described below.

\section{A. Simulation software environment}

Simulations have been performed using IDA-ICE 4.6, a commercial program for dynamic simulations of energy and comfort in buildings [29]. The program features equationbased modeling (NMF-language [34], [35] or Modelica language [36]) and is equipped with a variable time step differential-algebraic solver [37].

\section{B. Geometry of the simulated building}

The simulated indoor environment in Figure 1 represents the ground floor of a seven-storey university building in the KTH main campus in Stockholm. The rooms considered for our simulations are the labeled ones, and have different dimensions and use. The rooms have a Variable Air Volume (VAV) ventilation strategy where the mechanical ventilation airflow $u_{j}(k)$ varies depending on the current $\mathrm{CO}_{2}$ concentration in the room. In all rooms the ventilation is provided by a central fan active between 8:00 and 18:00. Room dimensions range from the $40 \mathrm{~m}^{2}$ of a small workshop (A:231) to the $130 \mathrm{~m}^{2}$ of a lecturing room (A:213), see 
Table I. The rooms have different occupancy and uses, which is reflected in the specific ventilation flows in the rooms; for instance, the project room (A:235) has more regular occupancy patterns than the conference hall (B:213), where periods of zero and high occupancies are alternated.

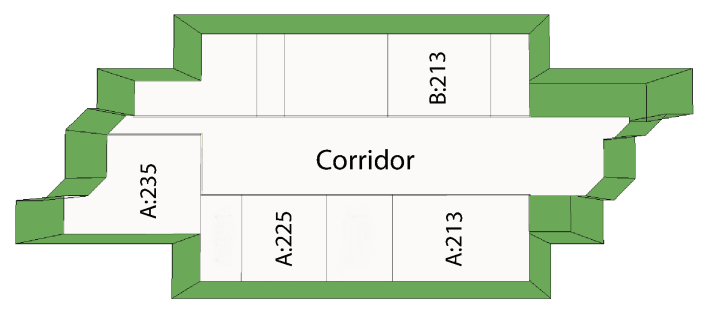

Fig. 1. Floor plan modeled in IDA-ICE.

\begin{tabular}{lccc}
\hline $\begin{array}{l}\text { Room } \\
\text { name }\end{array}$ & $\begin{array}{c}\text { Room size } \\
{\left[\mathrm{m}^{2}\right]}\end{array}$ & $\begin{array}{c}\text { Min air flow } \\
{\left[1 /\left(\mathrm{s} \mathrm{m}^{2}\right)\right]}\end{array}$ & $\begin{array}{c}\text { Max air flow } \\
{\left[1 /\left(\mathrm{s} \mathrm{m}^{2}\right)\right]}\end{array}$ \\
\hline A:235 & 125 & 0.6 & 2.16 \\
A:225 & 81 & 0.93 & 3.34 \\
A:213 & 130 & 0.58 & 2.1 \\
B:213 & 96 & 1.05 & 13.13 \\
\hline
\end{tabular}

TABLE I

ROOM FOOTPRINT, MINIMUM AND MAXIMUM ROOM MECHANICAL VENTILATION AIR FLOWS PER UNIT AREA. AIR FLOWS PER UNIT AREA ARE INDICATIVE OF THE NEED FOR VENTILATION AND THE LEVEL OF ACTIVITY IN THE ROOM.

\section{Simulation setup}

Room environment simulations were carried out for a period of two weeks between July 13 and July 26 in 2014. The climate file was a weather file for Bromma airport in Stockholm.

Air infiltrates through the windows and doors depending on the external wind speed and the air leakage area; Table II gathers the main infiltration parameters used in the simulations. The air tightness of the building is assumed to be 0.5 Air Changes per Hour (ACH) @ 50 Pa.

\begin{tabular}{lcc}
\hline $\begin{array}{l}\text { Room } \\
\text { name }\end{array}$ & $\begin{array}{c}\text { Windows surface } \\
{\left[\mathrm{m}^{2}\right]}\end{array}$ & $\begin{array}{c}\text { Air leakage area } \\
{\left[\mathrm{m}^{2}\right]}\end{array}$ \\
\hline A:235 & 10.6 & 0.015 \\
A:225 & 2.3 & 0.008 \\
A:213 & 3.4 & 0.014 \\
B:213 & 0 & 0.009 \\
\hline
\end{tabular}

TABLE II

TOTAL EXTERNAL WINDOWS AREA AND AIR LEAKAGE AREA IN THE ROOMS.

Each room has a different profile for the occupants; the level of activity of the occupants was set to 1.8 Metabolic Equivalent of Task (MET), corresponding to a light physical activity, such as typical office working conditions; $\mathrm{CO}_{2}$ emissions per person (parameter $g$ ), which is proportional to the activity, resulted in $15.4 \mathrm{mg}_{\mathrm{CO}_{2}} / \mathrm{s}$, corresponding to $8 \cdot 10^{-6} \mathrm{~m}_{\mathrm{CO}_{2}}^{3} / \mathrm{s}$.

\section{Validation of the data generation mechanism}

To assess the accuracy of the IDA-ICE physical model with respect to the real room dynamics, we compare measured and simulated $\mathrm{CO}_{2}$ data in Figure 2, under the same conditions of occupancy and ventilation levels [38]. The real data are collected from the laboratory room A:225 [30]. The two sets of measured and simulated data show that the physical model is capable to capture the main $\mathrm{CO}_{2}$ dynamics. The mismatch between the two curves is attributed to events whose effect, though minor, is not simple to account for; examples of such events are doors kept open and non-logged window openings.

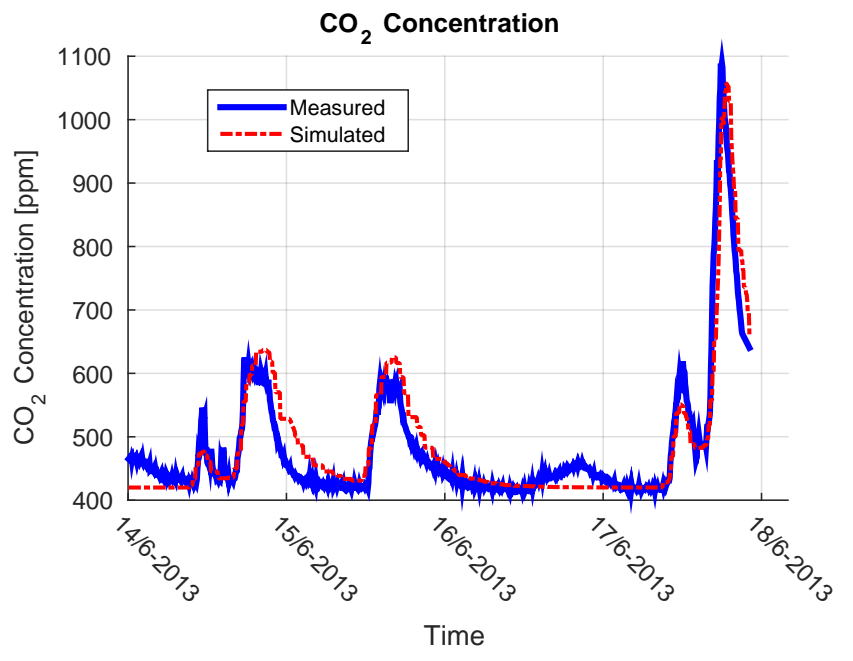

Fig. 2. Validation of the IDA-ICE model. $\mathrm{CO}_{2}$ levels from room measurements and from simulation are compared, from [38].

E. Assessing the single-room occupancy estimation algorithm

Here we compare the predictive capabilities of the singleroom model (7) against numerical representations of the rooms. This assessment is performed to check out whether the proposed model reproduces the internal and not accessible $\mathrm{CO}_{2}$ model of IDA-ICE. To this aim we:

1) collect the dataset $\mathcal{D}_{j}=\left\{c_{j}(k) u_{j}(k), o_{j}(k)\right\}_{k \in \mathcal{K}_{i}}$ for each room from the virtual room built in IDA-ICE;

2) add to $c_{j}(k)$ some artificial white Gaussian noise (whose variance is estimated from the real data used in SectionV-D and is equal to 35) and build the dataset

$$
\mathcal{D}_{j}:=\left\{y_{j}(k), u_{j}(k), o_{j}(k)\right\}_{k \in \mathcal{K}_{j}} ;
$$

3) identify the model, i.e., estimate the unknown part of $\boldsymbol{\theta}$ through the ML strategy discussed in Section IIB. This step corresponds to estimate the parameters $\widehat{\boldsymbol{\theta}}$ solving (15) and thus to obtain both the $\mathrm{CO}_{2}$ estimator $\widehat{y}_{j}(k ; \widehat{\boldsymbol{\theta}})$ through (14) and the occupancy estimator $\widehat{o}_{j}(k)$ through (17); 
The results for the estimated and measured $\mathrm{CO}_{2}$ for one day for the room A:225 are plotted in Figure 3, where it is possible to see that the proposed model is able to reproduce the $\mathrm{CO}_{2}$ generated by IDA-ICE. Realizations of the true occupancy and the estimated one for the same room is depicted in Figure 4. From Figure 4 it can be seen that the proposed occupancy estimator for a singleroom model can give accurate results in reproducing the true occupancy. In order to quantitatively evaluate the estimation capabilities of the single-room estimators, Table III provides some performance indexes for all rooms ${ }^{2}$. The Mean Square Error (MSE) of the estimations is small for all rooms and the algorithm has good detection of occupied rooms (small FNs).

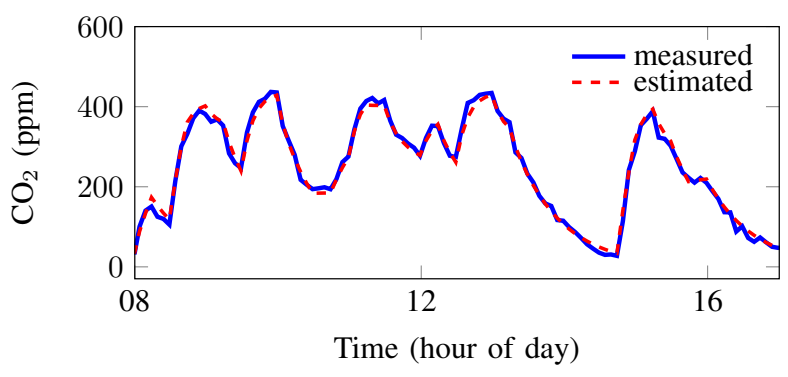

Fig. 3. Validation of model (7) against IDA-ICE for room A:225.

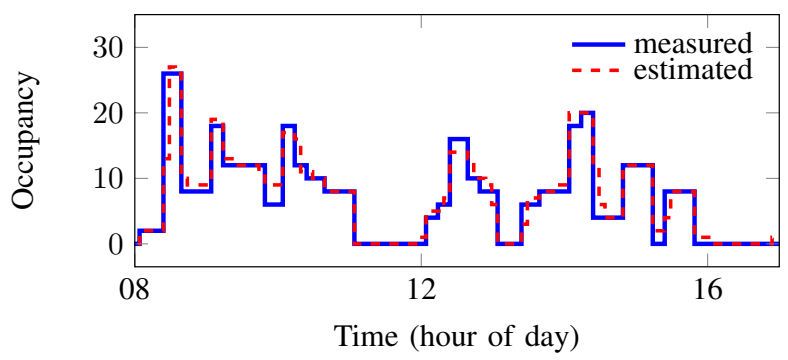

Fig. 4. Realizations of the true and estimated occupancy through the single-room estimator (17) for room A:213.

\begin{tabular}{ccccc}
\hline Room & MSE & Accuracy & FP & FN \\
\hline A:213 & 0.125 & 0.496 & 0.144 & 0.022 \\
A:225 & 0.247 & 0.563 & 0.280 & 0.000 \\
A:235 & 0.109 & 0.636 & 0.063 & 0.011 \\
B:213 & 0.075 & 0.750 & 0.047 & 0.021 \\
\hline
\end{tabular}

TABLE III

SUMMARY OF THE PERFORMANCE INDEXES OF THE COMPLETE SINGLE-ROOM ESTIMATORS.

\section{F. Assessing the multi-room model occupancy estimation algorithm}

To evaluate the effectiveness of the proposed multi-room occupancy estimator, we collect data from IDA-ICE for all

\footnotetext{
${ }^{2}$ The performance indices are described in Appendix I.
}

the rooms mentioned in Table I and we apply the occupancy estimator algorithm of Section IV.

In Figure 5, we provide the results of the occupancy estimation in one of the untrained rooms. It can be seen that the estimator is able to estimate the number of occupiers with fairly good precision, even though not as well as the single-room estimator. In order to have a better evaluation of the estimator, Table IV reports the performance indexes achieved by the estimator for all of the untrained rooms. The suggested multi-room estimator tends to have good ability on detecting occupancy levels in the rooms that are not instrumented with occupancy sensors. We noticed that there is a slight performance degradation in the estimated occupancy compared to the single-room case. This can be considered as a consequence of the assumptions made in (18), which do not hold for this simulation example (see Table I).

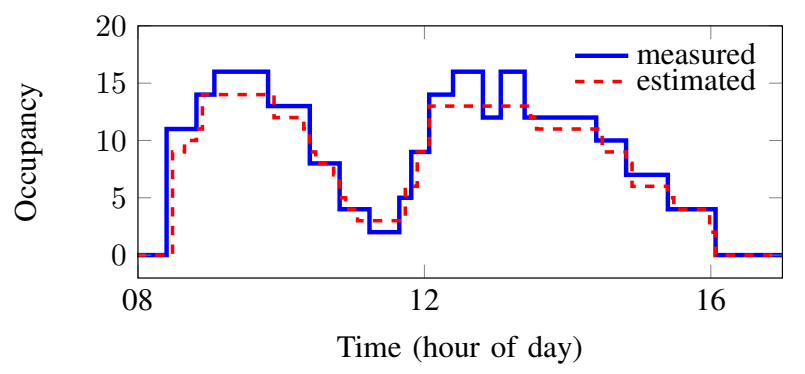

Fig. 5. Realizations of the true and estimated occupancy through the multiroom estimator for the untrained room A:213 when the model is trained on room A:225.

\begin{tabular}{cccccc}
\hline Trained Room & Untrained Room & MSE & Accuracy & FP & FN \\
\hline A:225 & A:235 & 0.179 & 0.413 & 0.364 & 0.001 \\
A:225 & A:213 & 0.232 & 0.276 & 0.399 & 0.000 \\
A:225 & B:213 & 0.104 & 0.489 & 0.062 & 0.012 \\
\hline
\end{tabular}

TABLE IV

SUMMARY OF THE PERFORMANCE INDEXES OF THE COMPLETE ESTIMATORS .

\section{CONCLUSIONS}

In this paper we have studied the problem of estimating the occupancy levels in buildings using available environmental and actuation signals. Our proposed method is centered on the $\mathrm{CO}_{2}$ dynamics which, starting from first principles, are modeled using a nonlinear gray-box model. The parameters of this model are identified on one of the rooms using a Maximum Likelihood (ML) approach. The resulting model is utilized to construct an occupancy estimator based on regularized deconvolution; this estimator is then adapted to other rooms of the building by exploiting the knowledge of the characteristics of the rooms and their relation with the room where the model is first identified. We have built a simulated environment where we have tested the estimation scheme, showing the effectiveness of the proposed scheme. 
A natural extension of the current work is the application of blind system identification techniques to the proposed scheme, so to remove the need of a training phase. More extensions may consider improving the estimations by using the knowledge of interconnection of the rooms and the locations of exits and entrances.

\section{REFERENCES}

[1] F. Oldewurtel, D. Sturzenegger, and M. Morari, "Importance of occupancy information for building climate control," Applied Energy, vol. 101, pp. 521-532, Jan. 2013.

[2] D. Sturzenegger, F. Oldewurtel, and M. Morari, "Importance of LongTerm Occupancy Information - A Validation with Real Occupancy Data," in Clima - RHEVA World Congress, 2013.

[3] O. Guerra Santin, L. Itard, and H. Visscher, "The effect of occupancy and building characteristics on energy use for space and water heating in Dutch residential stock," Energy and Buildings, vol. 41, no. 11, pp. 1223-1232, Nov. 2009.

[4] T. A. Nguyen and M. Aiello, "Energy intelligent buildings based on user activity: A survey," Energy \& Buildings, vol. 56, pp. 244-257, 2013.

[5] M. Gruber, A. Trüschel, and J.-O. Dalenbäck, "CO2 sensors for occupancy estimations: Potential in building automation applications," Energy and Buildings, vol. 84, pp. 548-556, Dec. 2014.

[6] C. Liao, Y. Lin, and P. Barooah, "Agent-based and graphical modelling of building occupancy," Journal of Building Performance Simulation, vol. 5, no. 1, pp. 5-25, Jan. 2012.

[7] J. Brooks, S. Goyal, R. Subramany, Y. Lin, T. Middelkoop, L. Arpan, L. Carloni, and P. Barooah, "An experimental investigation of occupancy-based control of commercial building climate," in IEEE Conference on Decision and Control, 2014.

[8] G. Liu, A. Dasu, and J. Zhang, "Review of Literature on Terminal Box Control, Occupancy Sensing Technology and Multi-zone Demand Control Ventilation (DCV)," U.S. Department of Energy, Tech. Rep. March, 2012.

[9] T. Labeodan, W. Zeiler, G. Boxem, and Y. Zhao, “Occupancy Measurement In Commercial Office Buildings For Demand-Driven Control Applications - A Survey And Detection System Evaluation," Energy and Buildings, vol. 93, pp. 303-3014, 2015.

[10] Shengwei Wang and Xinqiao Jin, "CO2-Based Occupancy Detection for On-Line Outdoor Air Flow Control," Indoor and Built Environment, vol. 7, pp. 165-181, 1998.

[11] D. Calì, P. Matthes, K. Huchtemann, R. Streblow, and D. Müller, "CO2 based occupancy detection algorithm: Experimental analysis and validation for office and residential buildings," Building and Environment, vol. 86, pp. 39-49, 2015.

[12] S. Wang, J. Burnett, and H. Chong, "Experimental Validation of CO2-Based Occupancy Detection for Demand-Controlled Ventilation," Indoor and Built Environment, vol. 8, no. 6, pp. 377-391, Nov. 1999.

[13] K. Weekly, N. Bekiaris-liberis, and A. M. Bayen, "Modeling and Estimation of the Humans' Effect on the CO 2 Dynamics Inside a Conference Room," arXiv, 2014.

[14] S. Kar and P. K. Varshney, "Accurate estimation of indoor occupancy using gas sensors," in International Conference on Intelligent Sensors, Sensor Networks and Information Processing. Ieee, Dec. 2009, pp. 355-360.

[15] A. Ebadat, G. Bottegal, D. Varagnolo, B. Wahlberg, and K. H. Johansson, "Estimation of building occupancy levels through environmental signals deconvolution," in ACM Workshop On Embedded Systems For Energy-Efficient Buildings, 2013.

[16] A. Ebadat, G. Bottegal, D. Varagnolo, B. Wahlberg, H. Hjalmarsson, and K. H. Johansson, "Blind identification strategies for room occupancy estimation," in European Control Conference, 2015.

[17] H. Ohlsson, L. Ratliff, R. Dong, and S. Sastry, "Blind identification via lifting," in Proc. of the 19th IFAC World Congress, 2014.

[18] G. Ansanay-Alex, "Estimating Occupancy Using Indoor Carbon Dioxide Concentrations Only in an Office Building: a Method and Qualitative Assessment," in REHVA World Congress on Energy efficient, smart and healthy buildings (CLIMA), 2013, pp. 1-8.

[19] S. Meyn, A. Surana, Y. Lin, S. M. Oggianu, S. Narayanan, and T. A. Frewen, "A Sensor-Utility-Network Method for Estimation of Occupancy in Buildings," in IEEE Conference on Decision and Control, 2009, pp. 1494-1500.
[20] F. Viani, A. Polo, F. Robol, G. Oliveri, P. Rocca, and A. Massa, "Crowd detection and occupancy estimation through indirect environmental measurements," in European Conference on Antennas and Propagation, 2014.

[21] Z. Yang, N. Li, B. Becerik-Gerber, and M. Orosz, "A non-intrusive occupancy monitoring system for demand driven HVAC operations," in ASCE Construction Research Congress, 2012, pp. 2001-2010.

[22] B. Ai, Z. Fan, and R. X. Gao, "Occupancy Estimation for Smart Buildings by an Auto-Regressive Hidden Markov Model," in American Control Conference, 2014, pp. 2234-2239.

[23] K. P. Lam, M. Höynck, B. Dong, B. Andrews, Y.-s. Chiou, D. Benitez, and J. Choi, "Occupancy detection through an extensive environmental sensor network in an open-plan office building," in IBPSA Conference, 2009, pp. 1452-1459.

[24] B. Dong, B. Andrews, K. P. Lam, M. Höynck, R. Zhang, Y.-S. Chiou, and D. Benitez, "An information technology enabled sustainability test-bed (ITEST) for occupancy detection through an environmental sensing network," Energy and Buildings, vol. 42, no. 7, pp. 1038-1046, July 2010.

[25] W. Kleiminger, C. Beckel, T. Staake, and S. Santini, "Occupancy Detection from Electricity Consumption Data," in Proceedings of the 5th ACM Workshop on Embedded Systems For Energy-Efficient Buildings - BuildSys'13. Rome, Italy: ACM Press, 2013, pp. 1-8.

[26] C. Liao and P. Barooah, "An Integrated Approach to Occupancy Modeling and Estimation in Commercial Buildings," in American Control Conference, 2010, pp. 3130-3135.

[27] L. Ljung, System identification - Theory for the user, 2nd ed. Prentice-Hall, 1999.

[28] H. A. Aglan, "Predictive model for $\mathrm{CO}_{2}$ generation and decay in building envelopes," Journal of applied physics, vol. 93, no. 2, pp. 1287-1290, 2003.

[29] EQUA. (2015, June) Equa simulations AB: IDA-ICE website.

[30] "Kth-hvac open testbed website http://hvac.ee.kth.se/," http://hvac.ee. kth.se/.

[31] A. Forsgren, P. E. Gill, and M. H. Wright, "Interior methods for nonlinear optimization," SIAM review, vol. 44, no. 4, pp. 525-597, 2002.

[32] C. Rojas and B. Wahlberg, "How to monitor and mitigate stair-casing in $\ell_{1}$ trend filtering," in 40th International Conference on Acoustics, Speech and Signal Processing (ICASSP), 2015.

[33] R. J. Tibshirani, The Solution Path of the Generalized Lasso. Stanford University, 2011.

[34] P. Sahlin, A. Bring, and E. F. Sowell, The neutral model format for building simulation. Citeseer, 1992.

[35] P. Sahlin, NMF Handbook: An Introduction to the Neutral Model Format. ASHRAE, 1996.

[36] P. Fritzson, Principles of object-oriented modeling and simulation with Modelica 2.1. John Wiley \& Sons, 2010.

[37] P. Sahlin, L. Eriksson, P. Grozman, H. Johnsson, A. Shapovalov, and M. Vuolle, "Whole-building simulation with symbolic DAE equations and general purpose solvers," Building and Environment, vol. 39, no. 8, pp. 949-958, 2004.

[38] R. Risuleo, M. Molinari, G. Bottegal, H. Hjalmarsson, and K. Johansson, "A benchmark for data-based office modeling: challenges related to $\mathrm{CO}_{2}$ dynamics," in Proc. of the 16th IFAC Symp. on System Identification (submitted), 2015.

\section{APPENDIX I \\ PERFORMANCE INDICES}

We report the performance indices that are used in Section $\mathrm{V}$ for evaluation of the proposed algorithm.

1) The mean square error

$$
\operatorname{MSE}(\widehat{\boldsymbol{o}}):=\frac{\|\widehat{\boldsymbol{o}}-\boldsymbol{o}\|_{2}^{2}}{\|\boldsymbol{o}\|_{2}^{2}},
$$

characterizing the relative estimation errors.

2) The accuracy

$$
\operatorname{Acc}(\widehat{\boldsymbol{o}}):=1-\frac{\|\mathbb{1}(\widehat{\boldsymbol{o}}-\boldsymbol{o})\|_{1}}{N},
$$


reporting how many times the estimates are perfect by means of the $\ell_{1}$ norm of the indicator function

$$
\mathbb{1}(\boldsymbol{x}):=\left\{\begin{array}{c}
\mathbb{1}(x(1)) \\
\vdots \\
\mathbb{1}(x(N))
\end{array} \quad \mathbb{1}(x(t)):= \begin{cases}1 & \text { if } x(t)>0 \\
0 & \text { otherwise }\end{cases}\right.
$$

3) The false positive / false negative occupancy detection rates

$$
\operatorname{FP}(\widehat{\boldsymbol{o}}):=\widehat{\beta}(0), \quad \mathrm{FN}(\widehat{\boldsymbol{o}}):=1-\widehat{\beta}(1),
$$

describing the ability of discriminating the presence / absence of occupants in terms of false positives (when the room is estimated to be occupied while it is not) and false negatives (when the room is estimated to be empty while it is not) by means of the empirical power function

$$
\widehat{\beta}(\theta):=\frac{1}{\left|\mathcal{N}_{\theta}\right|} \sum_{k \in \mathcal{N}_{\theta}} \mathbb{1}(\widehat{o}(k)),
$$

in its turn based on the definition of the sets

$$
\mathcal{N}_{\theta}:=\{t \text { s.t. } \mathbb{1}(o(k))=\theta\}, \quad \theta=\{0,1\},
$$

dividing the time indexes in the sets $\mathcal{N}_{0}$, for the $k$ 's for which the room was not occupied, and $\mathcal{N}_{1}$, for the $k$ 's

\begin{tabular}{|c|c|c|}
\hline parameter & description & unit \\
\hline$j \in \mathbb{N}_{+}$ & room index & adim. \\
\hline$t \in \mathbb{R}$ & time index (continuous) & adim. \\
\hline$k \in \mathbb{N}_{+}$ & time index (discrete) & adim. \\
\hline$o_{j}(k)$ & occupancy at time $k$ in room $j$ & adim. \\
\hline$g$ & $\begin{array}{l}\mathrm{CO}_{2} \text { generation rate per person } \\
\text { (assumed constant and known) }\end{array}$ & $\mathrm{m}_{\mathrm{CO}_{2}}^{3} / \mathrm{s}$ \\
\hline $\bar{c}_{j}(k)$ & $\begin{array}{l}\mathrm{CO}_{2} \text { concentration level at time } k \\
\text { in room } j\end{array}$ & ppm \\
\hline$c$ & $\begin{array}{l}\mathrm{CO}_{2} \text { concentration level of the air } \\
\text { injected by the ventilation system } \\
\text { (assumed constant and known) }\end{array}$ & ppm \\
\hline $\begin{array}{c}c_{j}(k):= \\
\bar{c}_{j}(k)-c\end{array}$ & $\begin{array}{l}\text { normalized } \mathrm{CO}_{2} \text { concentration } \\
\text { level at time } k \text { in room } j\end{array}$ & $\mathrm{ppm}$ \\
\hline$y_{j}(k)$ & noisy measurement of $c_{j}(k)$ & ppm \\
\hline$u_{j}(k) \in[0,1]$ & $\begin{array}{l}\text { actuation levels of the ventilation } \\
\text { system at time } k \text { in room } j\end{array}$ & adim., \% \\
\hline$\dot{Q}_{j}^{\max }$ & $\begin{array}{l}\text { nominal maximum airflow of the } \\
\text { ventilation system for room } j\end{array}$ & $\mathrm{~m}^{3} / \mathrm{s}$ \\
\hline$\dot{Q}_{j}^{\min }$ & $\begin{array}{l}\text { nominal minimum airflow of the } \\
\text { ventilation system for room } j\end{array}$ & $\mathrm{~m}^{3} / \mathrm{s}$ \\
\hline$\dot{Q}^{\text {leak }}$ & $\begin{array}{l}\text { leaking air flow (e.g., from win- } \\
\text { dows and doors; assumed constant } \\
\text { for each room) }\end{array}$ & $\mathrm{m}^{3} / \mathrm{s}$ \\
\hline$v_{j}$ & volume of room $j$ & $\mathrm{~m}^{3}$ \\
\hline $\mathcal{I} \subset \mathbb{N}_{+}$ & $\begin{array}{l}\text { set of rooms not instrumented } \\
\text { with occupancy sensors }\end{array}$ & adim. \\
\hline
\end{tabular}
for which the room was occupied.

\section{APPENDIX II \\ NotATION}

TABLE V

SUMMARY OF THE MOST IMPORTANT PARAMETERS. 